\title{
The Epidermal Growth Factor Receptor Increases Cytokine Production and Cutaneous Inflammation in Response to Ultraviolet Irradiation
}

\author{
Taghrid Bahig El-Abaseri, ${ }^{1,2}$ Brianna Hammiller, ${ }^{1}$ \\ Susan K. Repertinger, ${ }^{3}$ and Laura A. Hansen ${ }^{1,4}$ \\ ${ }^{1}$ Department of Biomedical Sciences, Creighton University, Omaha, NE 68178, USA \\ ${ }^{2}$ Medical Biochemistry Department, School of Medicine, Suez Canal University, Ismailia, Egypt \\ ${ }^{3}$ Department of Pathology, Creighton University Medical Center, Omaha, NE 68178, USA \\ ${ }^{4}$ Department of Biomedical Sciences, School of Medicine, Creighton University, 2500 California Plaza, Omaha, NE 68178, USA
}

Correspondence should be addressed to Laura A. Hansen; lhansen@creighton.edu

Received 30 April 2013; Accepted 30 May 2013

Academic Editors: D. T. Alexandrescu, B. Amichai, and C.-C. Yang

Copyright (C) 2013 Taghrid Bahig El-Abaseri et al. This is an open access article distributed under the Creative Commons Attribution License, which permits unrestricted use, distribution, and reproduction in any medium, provided the original work is properly cited.

\begin{abstract}
The epidermal growth factor receptor (EGFR) is activated in cutaneous keratinocytes upon ultraviolet (UV) exposure and has been implicated in ultraviolet-(UV-)induced inflammation and skin tumorigenesis. Egfr mutant mice and EGFR inhibitors were used to investigate the hypothesis that EGFR activation augments inflammation following UV irradiation. Topical treatment of mouse skin with the EGFR inhibitor AG1478 before UV exposure suppressed UV-induced erythema, edema, mast cell infiltration, and neutrophil infiltration. Genetic ablation of Egfr and EGFR inhibition by AG1478 also suppressed the increase in the proinflammatory cytokines tumor necrosis factor $\alpha$ (TNF- $\alpha$ ), interleukin- $1 \alpha$, KC (murine IL-8), and cyclooxygenase-2 (COX-2) after UV exposure of cultured keratinocytes. Finally, genetic ablation of inhibition of EGFR in cultured keratinocytes decreased p38 activation after UV, while inhibition of p38 kinase reduced COX-2 expression after UV. These data demonstrate that EGFR regulates multiple aspects of UV-induced inflammation and suggest activation of p38 kinase leading to increased COX-2 and cytokine expression as one mechanism through which it acts.
\end{abstract}

\section{Introduction}

Epidermal growth factor receptor (EGFR) signaling is involved in important aspects of cutaneous biology, including the regulation of epidermal proliferation, apoptosis, cell adhesion, and migration. For example, EGFR signaling appears to be important for such adaptive biologic processes as wound healing [1]. On the other hand, excessive EGFR signaling may participate in processes that are ultimately destructive to skin, such as in the skin's carcinogenic response to ultraviolet (UV) exposure [2-4].

Solar UV radiation is a major environmental hazard that generates reactive oxygen species, induces DNA damage, and leads ultimately to skin inflammation, photoaging, and cancer development [5]. Erythema and edema are the grossly visible signs of UV-induced inflammation in mammalian skin [6]. These changes are associated histologically with dermal infiltration of neutrophils, followed later by macrophages and mast cells [7]. These cellular events are accompanied or preceded by the release of a wide variety of proinflammatory mediators, including certain enzymes and cytokines. For example, following skin exposure to UV light, levels of the pro-inflammatory enzyme cyclooxygenase-2 (COX-2) are increased [8], which in turn leads to production of prostaglandin E2 (PGE2), a potent mediator of UV-induced skin erythema [9]. In addition to the activation of various enzymes in all nucleated cells in the skin, cells secrete several cytokines in response to UV exposure. UV-induced cytokines include interleukin-(IL-) 8 [10-12], IL- $1 \alpha[13,14]$, and TNF- $\alpha$ $[12,15]$. Release of cytokines in response to UV plays a central 
role in the autorecruitment and activation of inflammatory cells [16] as well as the production of matrix metalloproteases (MMPs) [17], contributing to the final pathological changes seen in chronic sun-damaged skin.

Since UV exposure activates EGFR indirectly through a mechanism involving reactive oxygen species inactivation of protein tyrosine phosphatase kappa [18], multiple EGFR-dependent signaling pathways may contribute to the physiological and histological effects seen in UV-irradiated skin. In particular, p38 mitogen-activated protein (MAP) kinase plays a critical role in regulating cellular responses to UV. For example, p38 kinase is activated in cultured keratinocytes [19] and in skin upon UV exposure [3, 19]. p38 kinase upregulates the expression of the pro-inflammatory cytokine IL-8 in keratinocytes following UV exposure [20]. Interestingly, inhibition of p38 kinase decreases UV-induced expression of KC (murine IL-8) [18], COX-2 [18, 21, 22], and PGE2 [21], thus lessening skin erythema [18].

While deregulated EGFR signaling in the skin in response to UV irradiation is implicated in epidermal hyperplasia, proliferation, apoptosis, and tumor formation [3,4], its modulation of the inflammatory response is not fully understood. Therefore, the current study was designed to investigate the role of EGFR signaling in UV-induced skin inflammation. We investigated the role of EGFR in the molecular mechanisms implicated in UVA/B-induced skin inflammation in vitro using Egfr-null and wild-type keratinocytes and an EGFR inhibitor, and in vivo using EGFR inhibitor-treated mouse skin. Our data showed that EGFR led to activation of p38 kinase, increased COX-2 levels, enhanced expression of the pro-inflammatory cytokines, and increased dermal infiltration of neutrophils and mast cells following acute exposure to UV.

\section{Methods}

2.1. Cell Culture. Primary keratinocytes were isolated from newborn CD-1 mouse skin or from $\mathrm{Egfr}^{-/-}$null mice on a CD-1 background, as described previously [23]. In brief, the skin was floated on trypsin (Invitrogen, Carlsbad, CA, USA) at $4^{\circ} \mathrm{C}$ overnight; the epidermis separated from the dermis; the epidermis was minced, triturated, and centrifuged in SMEM (Invitrogen) containing 8\% Chelex-treated (Bio-Rad, Hercules, CA, USA) fetal bovine serum (Gemini Bioproducts, Woodland, CA, USA). Keratinocytes grown to $70-80 \%$ confluence were exposed to either 200 or $600 \mathrm{~J} / \mathrm{m}^{2} \mathrm{UVA} / \mathrm{B}$ or were sham irradiated in a thin layer of phosphate-buffered saline containing $0.05 \mathrm{mmol} / \mathrm{L}$ calcium. Some keratinocytes were pretreated with $1 \mu \mathrm{mol} / \mathrm{L}$ AG1478 or $5 \mu \mathrm{mol} / \mathrm{L}$ PD169316 (Calbiochem, San Diego, CA, USA), dissolved in DMSO or DMSO alone 1 (PD169316) or 2 h (AG1478) before UV exposure, and refed fresh medium containing the inhibitor or vehicle alone immediately after UV exposure. For cytokine analysis, cell lysate suspended in TRAP ${ }_{\text {EZE }}$ CHAPS lysis buffer (Chemicon, Temecula, CA, USA) was analyzed using a Luminex instrument (Upstate Biotechnology, Charlottesville, VA, USA) according to the manufacturer's protocol.
2.2. Animals. The dorsal hair of mice was trimmed with electric clippers and a shaver at least one day prior to UV exposure. Some mice were topically treated or injected intraperitoneally (i.p.) with $150 \mathrm{mg} / \mathrm{kg}$ of AG1478 in DMSO or the vehicle DMSO alone $2 \mathrm{~h}$ prior to UV exposure using a protocol we have previously published [3]. FS40T12 sunlamps (Westinghouse, NJ, USA) emitted approximately $70 \%$ UVB, 30\% UVA, and 1\% UVC, with a total output of $1.46 \mathrm{~mW} / \mathrm{cm}^{2}$, as measured with radiometric photodetector probes (Oriel, Stratford, CT, USA). Skinfold thickness in agematched, UV-exposed homozygous female Tg.AC mice on an $\mathrm{FVB} / \mathrm{N}$ background was measured using calipers. Egfrnull and wild-type newborn mice on a CD-1 background were genotyped as described previously [24]. Erythema was assessed as the presence or absence of skin redness twentyfour hours following UV exposure. All animal experiments were performed with the approval and oversight of Creighton University's Institutional Care and Use Committee.

2.3. Immunofluorescence and Microscopy. Dorsal skin sections were fixed in formalin and stained with hematoxylin and eosin. Neutrophils were identified and counted in three representative microscopic fields on hematoxylin- and eosinstained sections with the investigator blinded as to the identity of the samples. Mast cells were identified and similarly counted following immunofluorescence with an antitryptase antibody (Cell Signaling) and DAPI (Vector Labs) to identify the nuclei.

2.4. Immunoblotting. Cells were lysed in buffer containing $10 \mathrm{mM}$ Tris pH 7.4, $150 \mathrm{mM} \mathrm{NaCl}, 10 \%$ glycerol, $1 \%$ Triton $\mathrm{X}-100,1 \mathrm{mM}$ EDTA, complete protease inhibitor (Roche, Germany), $1 \mathrm{mM} \mathrm{Na} \mathrm{VO}_{4}, 1.5 \mathrm{M}$ EGTA, and $10 \mathrm{M}$ NaF. Protein levels were quantitated using the BioRad assay (BioRad, Hercules, CA, USA). Immunoblotting with antibodies recognizing COX-1 (Cayman Chemical, Ann Arbor, MI 48108), COX-2 (Cayman), phospho-p38 kinase (Cell Signaling, Beverly, MA, USA), p38 kinase (Cell Signaling), phospho-ATF2 (Cell Signaling), and actin (Sigma, St.Louis, MO, USA) was performed using the appropriate horseradish peroxidaseconjugated secondary antibody (Cell Signaling) and chemiluminescence reagents (Pierce, Rockford, IL, USA). Densitometry was performed using 1DScan software (Scanalytics, Fairfax, VA, USA).

\section{Results}

3.1. Inhibition of EGFR Decreases UV-Induced Erythema and Edema. To investigate the role of EGFR on the acute inflammatory response, we examined two macroscopic signs of acute UV-induced skin damage, erythema and edema, in mice topically treated with the EGFR inhibitor AG1478 or with the vehicle alone two hours prior to exposure to $10 \mathrm{~kJ} / \mathrm{m}^{2} \mathrm{UVA} / \mathrm{B}$. Within a few hours following a single UV exposure, skin erythema developed in both EGFR inhibitorand vehicle-treated mice (data not shown). Twenty-four hours after UV, vehicle-treated skin showed severe skin redness (Figure 1(a), left panels). In contrast to vehicle-only 

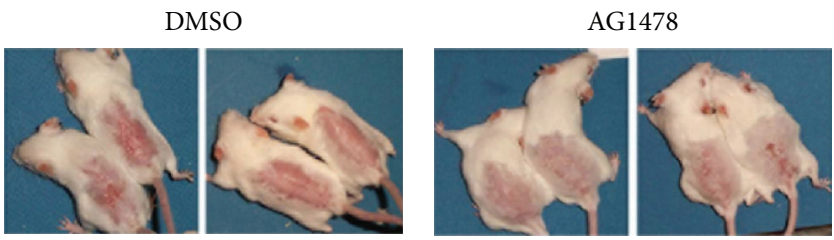

(a)

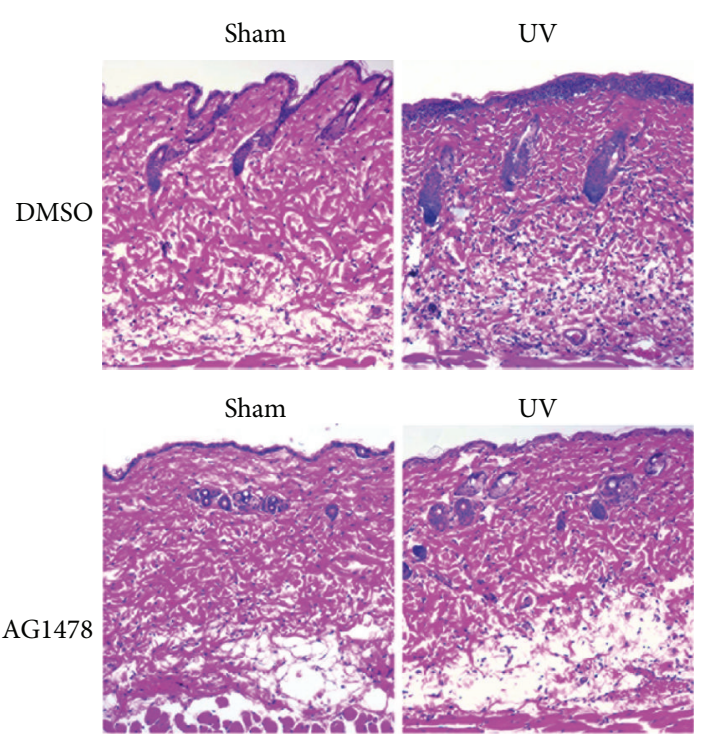

(c)

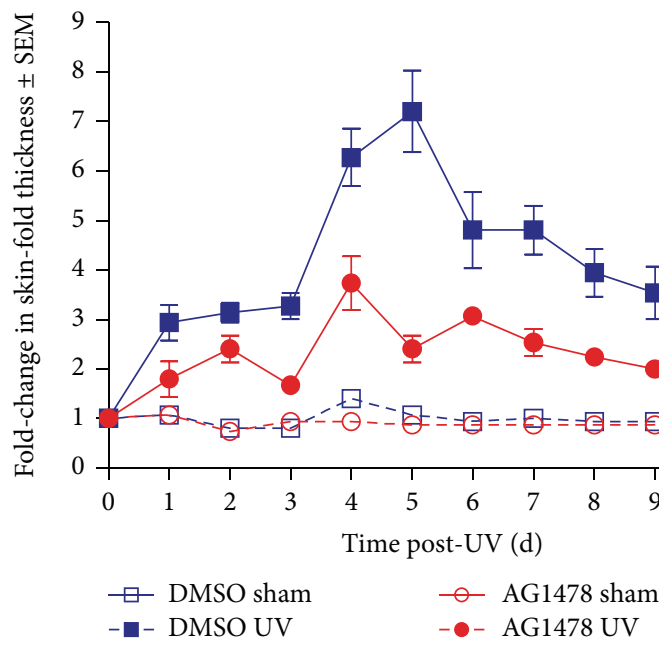

(b)

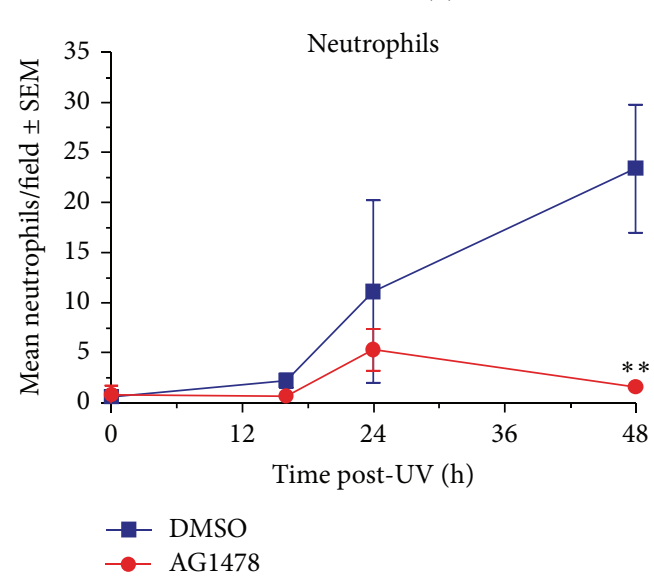

(d)

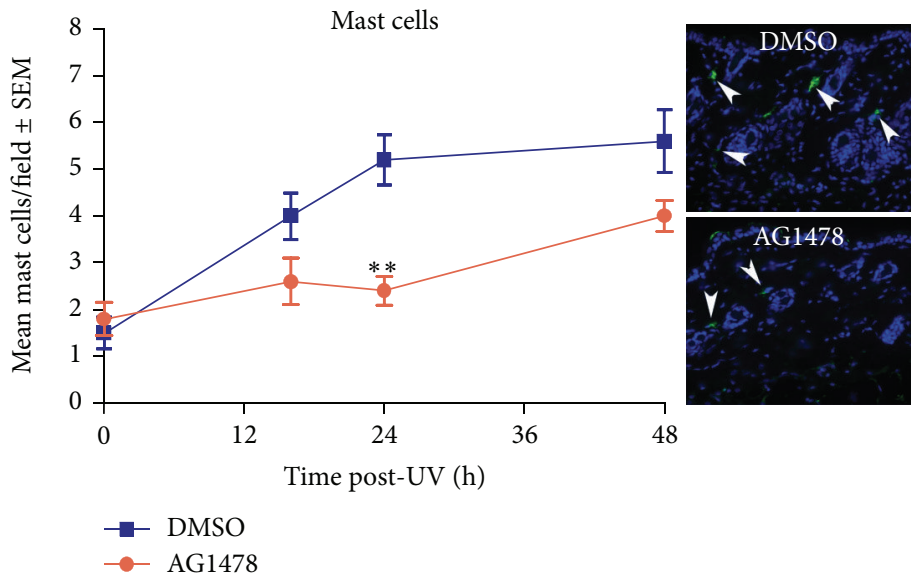

(e)

FIGURE 1: EGFR inhibition results in less acute UV-induced skin injury. Mice were treated topically with AG1478 or vehicle alone and exposed to $10 \mathrm{~kJ} / \mathrm{m}^{2}$ UV or sham-irradiated. (a) Mice treated with AG1478 (right) or vehicle (left) were photographed at $24 \mathrm{~h}$ following UV exposure. (b) Skinfold thickness was measured daily following UV. Mean \pm standard error is shown. $N=10$ mice. ${ }^{*}$ Indicates a significant difference compared to the vehicle-treated and sham-irradiated group between 1 and $11 \mathrm{~d}$ after irradiation, ${ }^{* *}$ significant compared to the vehicle-treated and UV-exposed group between 3 and $9 \mathrm{~d}$ after UV, or ${ }^{* * *}$ significant compared to the sham-irradiated groups at $2 \mathrm{~d}$ and between 4 and $8 \mathrm{~d}$ after UV, using two-way ANOVA, where $P \leq 0.05$. (c) Hematoxylin- and eosin-stained sections revealed increased dermal cellularity in UVexposed and vehicle treated skin $48 \mathrm{~h}$ after UV (200x magnification shown). (d) Neutrophils were counted in at least three 4x microscopic fields in hematoxylin- and eosin-stained sections with the investigator blinded as to the identity of the samples. The mean number of neutrophils per field \pm standard error is shown. $N=3$ mice. (e) Mast cells were counted in 20x microscopic fields in tryptase-stained sections with the investigator blinded as to the identity of the samples. The mean number of tryptase-positive cells per field \pm standard error is shown on left and representative images from UV-irradiated skin at the $24 \mathrm{~h}$ time point. $N=3$ mice. ${ }^{* *}$ Indicates a significant difference compared to the vehicle-treated control. 
treated skin, a single topical application of AG1478 prior to UV irradiation markedly lessened the severity of erythema when mice were examined at the same time point (Figure 1(a), right panels). Similar observations were made in experiments in which the inhibitor was injected intraperitoneally rather than topically applied (data not shown), indicating that the decreased erythema after AG1478 did not result from a sunblocking effect.

UV-associated edema, as measured by skin-fold thickness, was greater in both EGFR inhibitor- and vehicletreated mouse skin when compared with sham-irradiated skin (Figure 1(b)). Skin-fold thickness in both groups was greatest at 4-5 days after UV exposure. AG1478 application resulted in less than half as much edema at four and five days after irradiation with less edema in inhibitor-treated skin for the duration of the $11 \mathrm{~d}$ experiment (Figure 1(b)). Intraperitoneal injection of AG1478 prior to UV irradiation similarly suppressed the increase in skin-fold thickness (data not shown). Thus, EGFR activation correlates positively with the macroscopic signs of skin edema and erythema following UV exposure.

3.2. Inhibition of EGFR Decreases UV-Induced Neutrophil and Mast Cell Infiltration. To further elucidate the role of EGFR in the inflammatory response to UV, the skin was examined histologically for signs of inflammation after UV irradiation. Hematoxylin- and eosin-stained skin sections from vehicle treated and UV-exposed skin revealed increased dermal cellularity, consistent with infiltration of inflammatory cells, when compared to the sham-irradiated control (Figure 1(c), top). In contrast, inhibition of EGFR suppressed this response (Figure 1(c), bottom). Inhibition of EGFR resulted in less damage to the epidermis and reduced dermal cellularity, consistent with decreased inflammation in the skin (Figure 1(c), bottom).

To further investigate the effect of inhibition of EGFR on inflammation, neutrophils and mast cells were examined. Neutrophils play roles in acute inflammation following UV exposure and contribute to angiogenesis following UV through the release of human leukocyte elastase that degrades dermal elastin [25]. Neutrophil numbers were quantified in hematoxylin- and eosin-stained skin sections from vehicleand inhibitor-treated UV- and sham-irradiated skin. While the baseline number of neutrophils in sham-irradiated controls was not significantly different from that of inhibitortreated skin, a single UV exposure increased neutrophil skin infiltration to a greater extent in the vehicle-treated mice (Figure 1(d)). A significant increase in neutrophil number was detected in vehicle-treated skin as early as $16 \mathrm{~h}$ after UV-irradiation (Figure 1(d)). The number of neutrophils continued to increase in vehicle treated skin to a maximum of 23.4 neutrophils per microscopic field at $48 \mathrm{~h}$ (Figures 1(c)-1(d)). In contrast, in inhibitor-treated skin neutrophils did not increase until $24 \mathrm{~h}$ after UV and peaked at only 5.3 neutrophils per microscopic field at this time, less than half of the number in the vehicle-treated group. By $48 \mathrm{~h}$ neutrophil number declined to 1.6 neutrophils per field in the inhibitor treated mice, less than $10 \%$ of that in the vehicle treated skin (Figures 1(c)-1(d)).
In addition to neutrophils, mast cells participate in the UV-induced skin inflammatory response [26-28]. For example, mast cells are believed to contribute to UV-induced sun damage through the release of potent pro-inflammatory mediators, including histamine, leukotrienes, and tumor necrosis factor- $\alpha$ [28]. The effect of EGFR inhibition of mast cells was examined in tryptase-stained skin sections. Shamirradiated skin pretreated with AG1478 showed a similar number of mast cells when compared to vehicle-treated skin (Figure 1(e)). UV exposure increased mast cell number in vehicle-treated skin by $18 \mathrm{~h}$, with an even greater increase at 24 and $48 \mathrm{~h}$ after UV (Figure 1(e)). In contrast, mast cell numbers in inhibitor treated skin did not significantly increase in the first $24 \mathrm{~h}$ after UV (Figure 1(e)). At $24 \mathrm{~h}$ after UV, the number of mast cells in inhibitor-treated skin was less than half that seen in the sham-irradiated skin (Figure 1(e)). By $48 \mathrm{~h}$ after UV, inhibitor-treated skin did have increased numbers of mast cells although they were still fewer than those in the vehicle-treated skin. These data indicate that UV-dependent EGFR activation regulates mast cell and neutrophil infiltration within the skin.

3.3. EGFR Activation in Response to UV Regulates the Expression of $I L-1 \alpha, T N F-\alpha$, and KC. To determine whether pharmacological inhibition of EGFR would affect cytokine production following UV irradiation, cultured keratinocytes were pretreated with the EGFR inhibitor AG1478 and UV exposed. Since EGFR phosphorylation was detected in keratinocytes 5 minutes following exposure to a range of UV exposures from 100 to $600 \mathrm{~J} / \mathrm{m}^{2}$ of UV (data not shown), cells were irradiated with $600 \mathrm{~J} / \mathrm{m}^{2} \mathrm{UV}$ and cytokine levels were measured in both cell lysate and in media $16 \mathrm{~h}$ following UV exposure or sham-irradiation (Figure 2). KC (the murine IL-8 homolog) and IL-1 $\alpha$ were detectable in both lysate and media of sham irradiated cells while TNF- $\alpha$ was only detectable in the cell lysate. No significant differences between shamirradiated and vehicle or inhibitor-treated keratinocytes were detected (Figure 2, white bars). As expected, UV exposure enhanced production of TNF- $\alpha$, KC, and IL- $1 \alpha$ cytokines in keratinocytes and in keratinocyte-conditioned media (Figure 2, black bars compared to white bars). Cells pretreated with the EGFR inhibitor had reduced levels of TNF- $\alpha, \mathrm{KC}$, and IL- $1 \alpha$ in both conditioned media and of TNF- $\alpha$ in cell lysates $16 \mathrm{~h}$ following UV exposure when compared with DMSO-treated and UV-exposed keratinocytes (Figure 2).

To verify the involvement of EGFR in cytokine expression following UV irradiation, Egfr-null and wild-type keratinocytes were exposed to $600 \mathrm{~J} / \mathrm{m}^{2}$ or sham-irradiated and the levels of TNF- $\alpha, \mathrm{KC}$, and IL-1 $\alpha$ were measured in cell lysate and in media $16 \mathrm{~h}$ later (Figure 3). Cytokine levels tended to be higher in the controls of this experiment than in the inhibitor experiment, suggesting a suppression of cytokine levels by the vehicle DMSO (Figure 3 compared to Figure 2). Genetic ablation of Egfr resulted in a trend toward decreased baseline levels of the KC and IL- $1 \alpha$ when compared to Egfr wild-type cells in both lysate and medium (Figure 3, white bars). Following UV exposure, increased levels of TNF$\alpha, \mathrm{KC}$, and IL- $1 \alpha$ were measured in wild-type medium, 

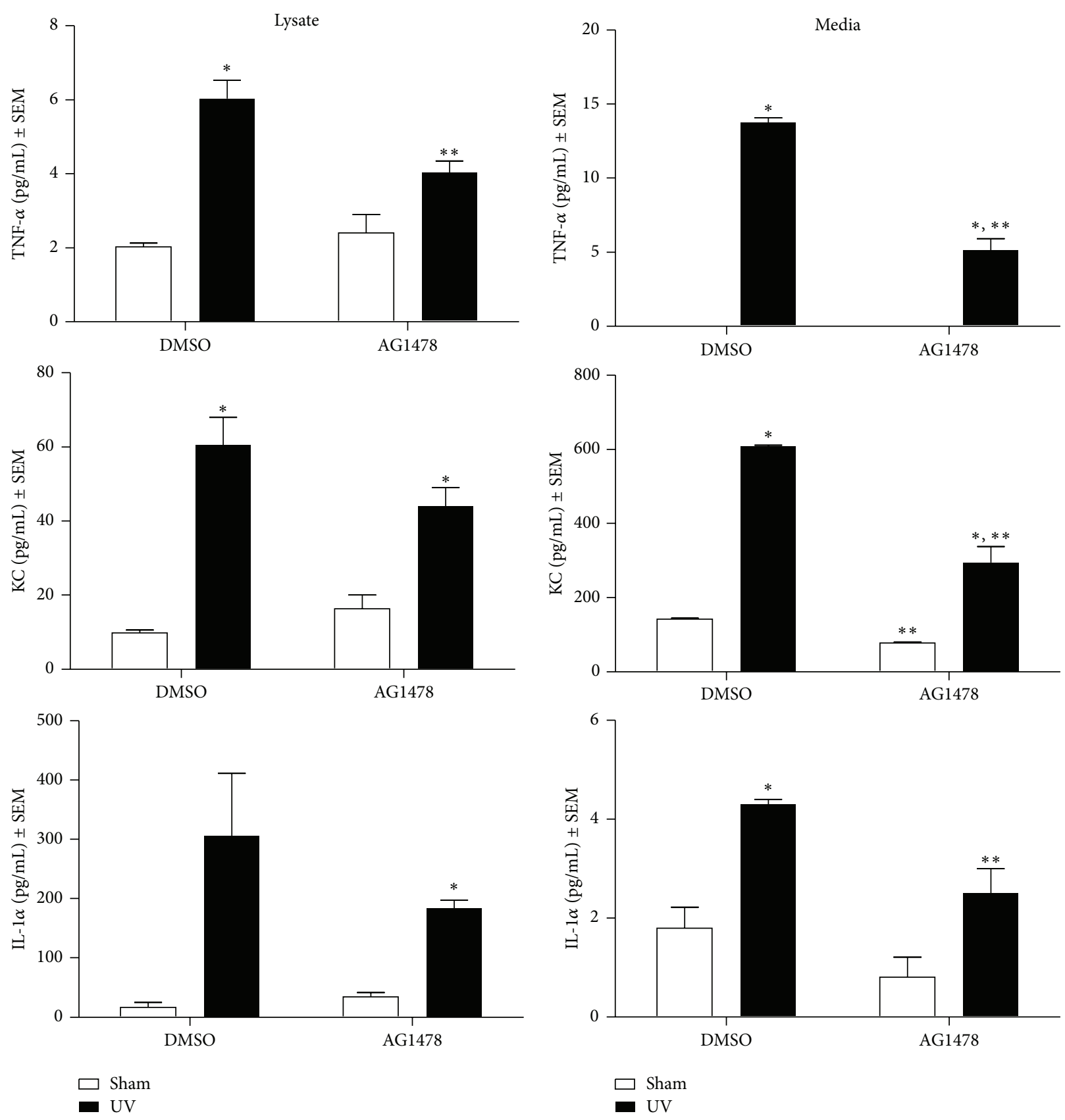

(a)

(b)

FIGURE 2: EGFR inhibition reduces TNF- $\alpha$, KC, and IL- $1 \alpha$ in keratinocytes following UV exposure. Subconfluent keratinocytes were treated with $1 \mu \mathrm{M}$ AG1478 or the vehicle DMSO. Cells were exposed to $600 \mathrm{~J} / \mathrm{m}^{2} \mathrm{UV}$ and cell lysate (left panel) and media (right panel) were prepared at $16 \mathrm{~h}$ for cytokine analysis using Luminex technology. Data from at least two different experiments, with at least three dishes per group, are presented as mean \pm standard error. ${ }^{*}$ Mean is significantly different from the corresponding sham-irradiated control or ${ }^{* *}$ significantly different from the corresponding vehicle-treated group, using a Student's $t$-test, where $P \leq 0.05$.

and of TNF- $\alpha$ and IL-1 $\alpha$ in $\mathrm{Egfr}^{-/-}$medium (Figure 3). The magnitude of the increase in TNF- $\alpha$, IL- $1 \alpha$, and $\mathrm{KC}$ was greater in the Egfr wild-type conditioned medium. $\mathrm{KC}$ was the only cytokine increased in wild-type lysate, while both KC and IL-1 $\alpha$ were increased in Egfr-null lysate. Taken together, our results suggest that EGFR signaling contributes to increased TNF- $\alpha, \mathrm{KC}$, and IL- $1 \alpha$ levels in sham- and UVirradiated keratinocytes.

3.4. COX-2 Expression in Keratinocytes Is Regulated by EGFR. UV exposure induces epidermal expression of COX-2, an important enzyme that regulates cytokine production and 

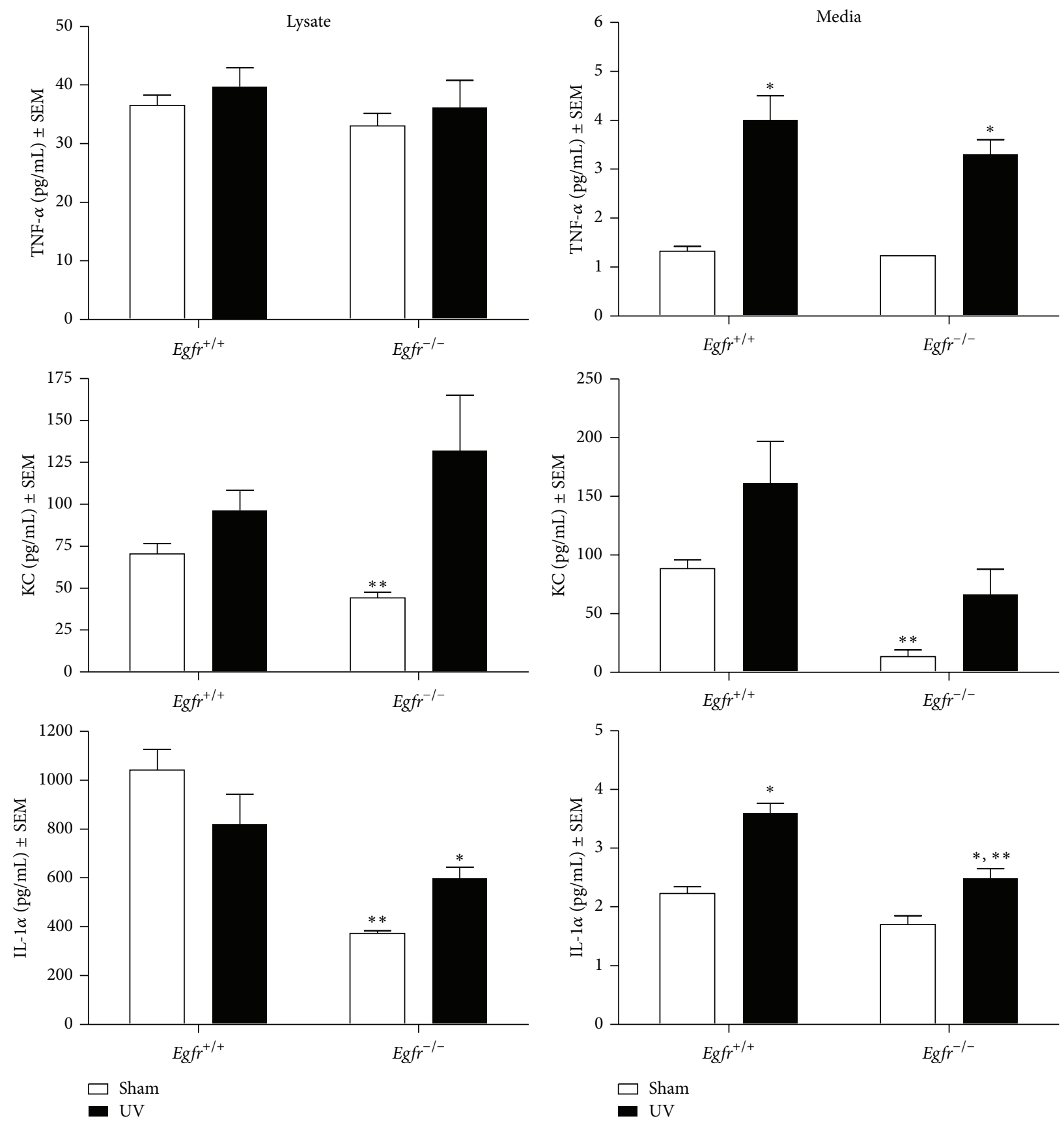

(a)

(b)

FIGURE 3: Genetic deletion of Egfr reduces TNF- $\alpha$, KC, and IL- $1 \alpha$ in keratinocytes following UV exposure. Subconfluent Egfr-null and wildtype keratinocytes were exposed to $600 \mathrm{~J} / \mathrm{m}^{2} \mathrm{UV}$ irradiation and cell lysate (left panel) and media (right panel) were harvested for cytokine analysis at $16 \mathrm{~h}$ using a Luminex instrument. $N \geq 4$ dishes. ${ }^{*}$ Mean is significantly different from the corresponding sham-irradiated control or $^{* *}$ significantly different from the corresponding wild-type group, using a Student's $t$-test, where $P \leq 0.05$.

inflammation $[8,21,22]$. In addition, topical inhibition of COX-2 effectively inhibits UVB-mediated inflammation [29]. To determine whether EGFR contributed to the UVinduced increase in cytokine levels through a mechanism involving COX-2, we examined levels of COX-2 following UV exposure in keratinocytes treated with AG1478 or the vehicle alone and in Egfr-null and wild-type keratinocytes. COX-2 protein was increased more than threefold by $16 \mathrm{~h}$ following UV exposure of vehicle-treated keratinocytes (Figure 4(a)). Inhibition of EGFR had no effect on COX-2 in shamirradiated cells (Figure 4(a)). However, pretreatment with the EGFR inhibitor largely prevented the UV-induced increase 


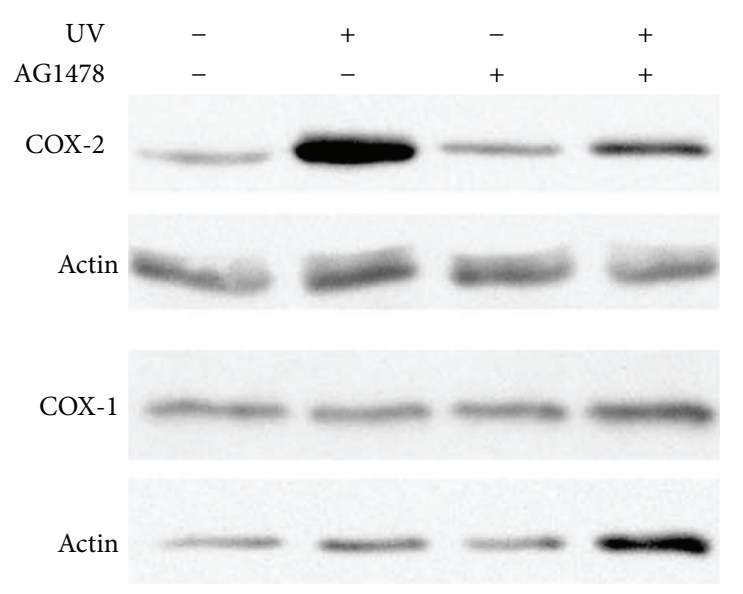

(a)

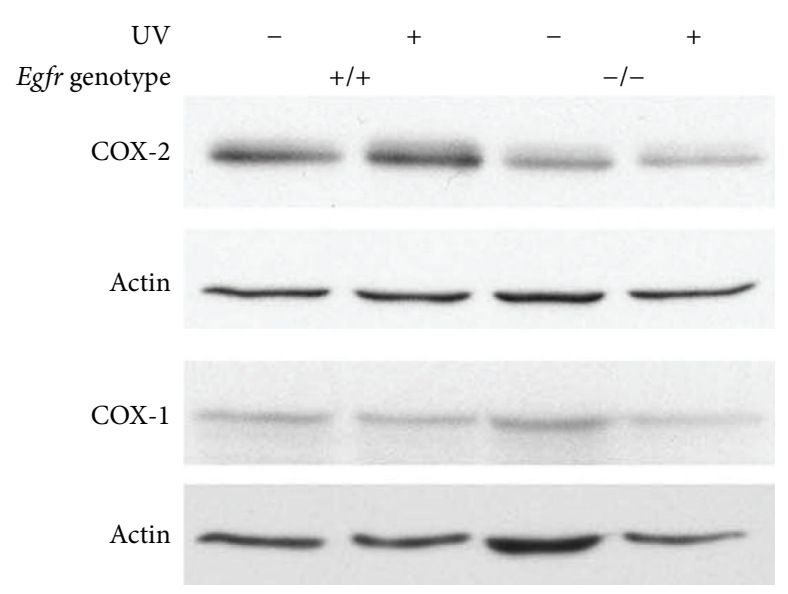

(b)

FIGURE 4: EGFR increases COX-2 levels in keratinocytes following UV exposure. (a) Subconfluent keratinocytes were treated with $1 \mu \mathrm{M}$ AG1478 or vehicle $2 \mathrm{~h}$ prior to $200 \mathrm{~J} / \mathrm{m}^{2}$ UV irradiation or sham-irradiation. (b) Subconfluent Egfr-null and wild-type keratinocytes were UV-exposed $\left(200 \mathrm{~J} / \mathrm{m}^{2}\right)$ or sham-irradiated. (a)-(b) Sixteen hours after irradiation, protein lysate was prepared. Samples were immunoblotted with the indicated antibodies. Data are representative of three experiments performed with similar results.

in COX-2 (Figure 4(a)). As expected, COX-1, the constitutive form, was not increased by UV and inhibition of EGFR did not alter COX-1 levels (Figure 4(a)).

To determine whether genetic ablation of Egfr would produce similar effects on COX-2, Egfr-null and wild-type cells were similarly exposed and COX proteins assessed. Baseline levels of COX-2 expression were lower in Egfrnull keratinocytes in comparison with wild-type control cells (Figure 4(b)). UV exposure enhanced COX-2 expression only slightly in wild type keratinocytes and not at all in Egfrnull keratinocytes (Figure 4(b)). Levels of COX-1 were not affected by deletion of Egfr or UV irradiation. Thus, although slightly different results were obtained using the two models for blockade of EGFR signaling, both sets of experiments are consistent with EGFR upregulation of COX-2 after UV exposure.

3.5. p38 Kinase Activation in Keratinocytes Is Dependent on UV-Induced EGFR Activation. Numerous studies have shown that p38 kinase is activated by UV in both human keratinocyte cell lines [21] and in mouse skin [18] Topical inhibition of p38 kinase effectively inhibits UVB-mediated inflammation [18, 30]. In addition, EGFR regulates in vivo levels of phosphorylated p38 kinase following UV exposure [3]. To explore the effects of EGFR inhibition on the activation of p38 kinase as a mechanism for COX-2 regulation, wild-type keratinocytes were treated with AG1478 prior to UV exposure or sham irradiation. p38 kinase activity was assessed indirectly by examining p38 kinase phosphorylation on immunoblot. Fifteen minutes following UV exposure, levels of the phosphorylated, active form of p38 kinase were increased in vehicle-treated and UV-exposed cells when compared to sham-irradiated controls (Figure 5(a)). Inhibition of EGFR reduced the UV-stimulated phosphorylation of p38 kinase (Figure 5(a)). In the parallel experiment using Egfr wild-type and null keratinocytes, the increase in p38 kinase activity in response to UV was less striking in the control genotype (Figure 5(b)), when compared to the vehicle-treated keratinocytes of Figure 5(a). However, genetic deletion of Egfr further suppressed p38 kinase phosphorylation in response to UV (Figure 5(b)). Thus, activation of p38 kinase by UV is partially dependent on EGFR signaling.

3.6. Inhibition of p38 Kinase Reduced EGFR-Induced COX2 Expression in Response to UV Irradiation. To investigate whether $\mathrm{p} 38$ kinase-dependent signaling is required for UVinduced expression of COX-2, we used the p38 kinase inhibitor PD169316. UV exposure activated p38 kinase as reflected by the increased phosphorylation of the p38 kinase substrate activating transcription factor-2 (ATF2) [30] (Figure 5(c)). Egfr wild-type keratinocytes were treated with $5 \mu \mathrm{M}$ inhibitor for $1 \mathrm{~h}$ before exposure to UV, with continuing incubation in the presence of the inhibitor. Inhibition of p38 kinase reduced the activation of p38 as detected by reduced phosphorylated ATF2 when compared to sham-irradiated levels at 15 minutes following UV (Figure 5(c)). In addition, inhibition of $\mathrm{p} 38$ kinase prior to UV exposure reduced COX2 in Egfr wild-type cells at $16 \mathrm{~h}$ (Figure 5(d)), demonstrating that p38 kinase activity is necessary for full expression of COX-2 after UV irradiation.

\section{Discussion}

In this study, we examined the direct involvement of EGFR signaling in mediating skin inflammation upon UV exposure. We found that inhibition of EGFR suppressed UV-induced edema and erythema in mouse skin. Similarly, neutrophil and mast cell infiltration of the skin following UV exposure were also suppressed with blockade of EGFR. Using cell culture models, we further examined cytokine levels in EGFR inhibitor-treated and Egfr-null keratinocytes. Some differences in the response to UV were detected between 


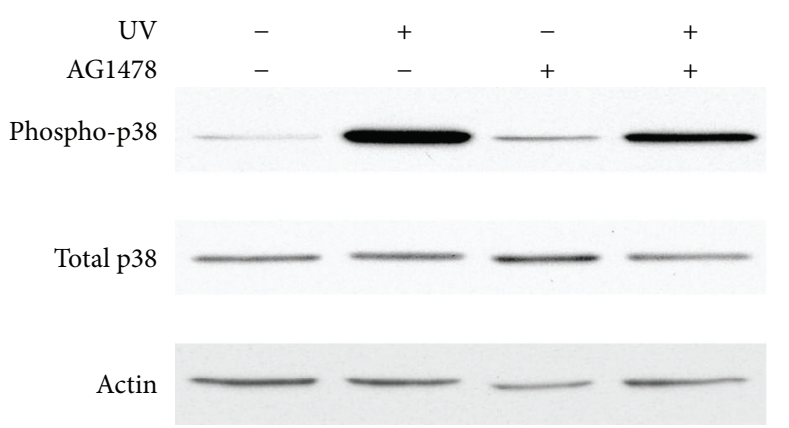

(a)

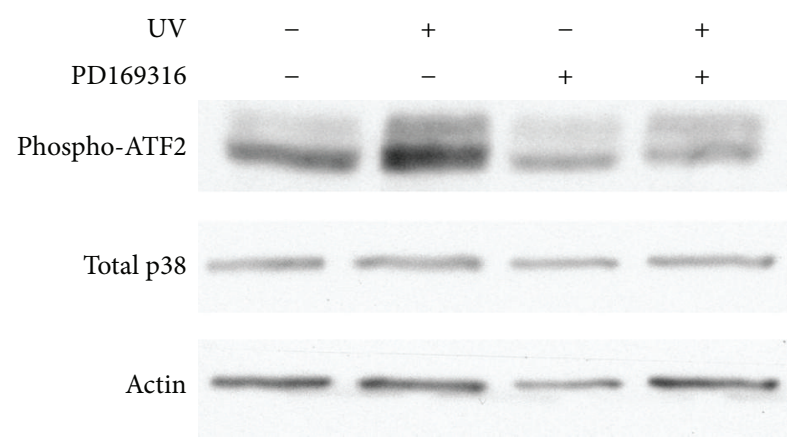

(c)

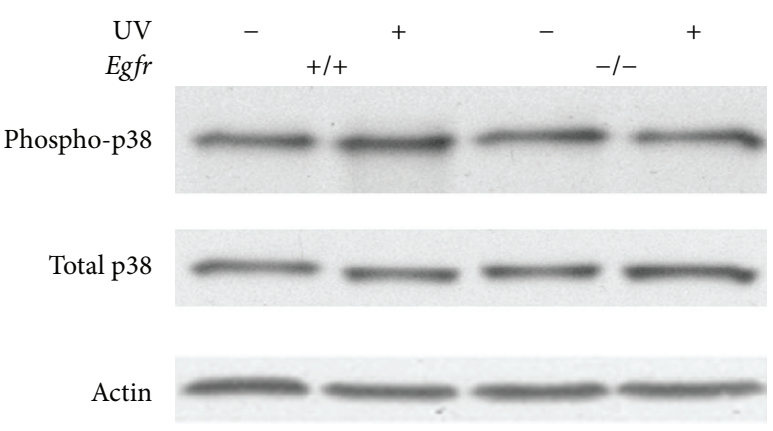

(b)

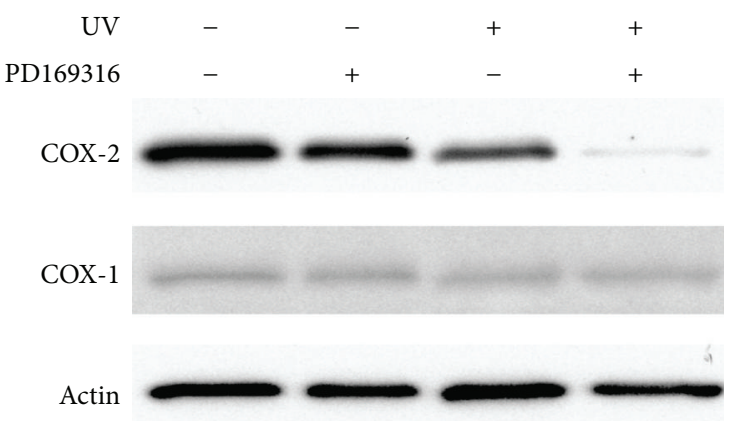

(d)

FIGURE 5: Inhibition of p38 kinase reduces COX-2 in UV-irradiated keratinocytes. Subconfluent wild-type keratinocytes (a)-(d) or Egfr-null keratinocytes (b) were treated with AG1478 (a), PD169316 (c), (d), or vehicle alone (a), (c), (d), followed by exposure to $200 \mathrm{~J} / \mathrm{m}^{2}$ or sham irradiation. Fifteen minutes (a)-(c) or $16 \mathrm{~h}$ later (d), protein lysate was prepared. Samples were immunoblotted with the indicated antibodies. Data are representative of three experiments performed with similar results.

the inhibitor and genetic models, suggesting an effect of the vehicle DMSO. Taken together, however, these experiments demonstrated that decreased levels of TNF- $\alpha$, KC (mouse homolog of IL-8), IL-1 $\alpha$, and COX-2 in response to UV resulted from abrogation of EGFR in mouse keratinocytes. Inhibition of the EGFR-activated p38 kinase similarly suppressed COX-2 levels in UV-irradiated keratinocytes, suggesting EGFR activation of p38 kinase as a potential mechanism for the increases in COX-2 and cytokines associated with UV-induced inflammation.

The inflammatory response to UV is likely the result of both EGFR-dependent and EGFR-independent signaling pathways. MAPK transduction pathways are involved in modulating cytokine production in mouse skin inflammatory response to chemical irritants [31, 32]. In particular, p38 kinase is activated by phosphorylation in keratinocytes in vitro [21] and in vivo [33] in response to UV. Therefore, we investigated whether EGFR regulates p38 kinase activation in response to UV. Our analyses revealed that p38 kinase is activated in response to UV in manner partially dependent on EGFR. In addition, our data show that COX-2 expression is largely dependent on EGFR-mediated p38 kinase activation. Previous studies using murine models have demonstrated that topical inhibition of COX-2 after UV exposure inhibited inflammation $[29,34]$, presumably through decreased production of PGE2 and vascular endothelial growth factor (VEGF), reducing erythema and edema $[8,27,29,35,36]$. Interestingly, COX-2 is highly expressed in murine and human nonmelanoma UV-induced skin tumors, while inhibition of COX-2 appears to decrease such tumor formation [37-39]. Furthermore, since some authors have reported that COX-2-derived PGE2 synthesis is a key event of skin tumor promotion in response to UV [34], COX-2 suppression resulting from EGFR inhibition is worthy of further investigation.

In addition to the EGFR-dependent up-regulation of COX-2 through p38 kinase documented here, additional EGFR-dependent mechanisms regulating inflammation have been documented. For example, activated MEK1-ERK signaling contributes to increased IL- $1 \alpha$ production [31] and TNF- $\alpha$ expression [32] in keratinocytes. Similarly, EGFR signaling through MEK1/2 and p38 kinase synergizes with IL- $1 \alpha$ in the skin innate immune response by enhancing the production of antibacterial peptides in normal skin and chronic inflammatory diseases like psoriasis [40]. In addition, an immunomodulatory role of EGFR was suggested by the increased expression of granulocyte/macrophage colonystimulating factor (GM-CSF), a pro-inflammatory cytokine in mouse and human skin [31,32, 41], although this finding has not been investigated in the context of UV-induced skin inflammation.

Skin infiltration with neutrophils and mast cells is a hallmark of various skin inflammatory disorders and in that of UV-induced inflammation [27, 42, 43]. Our findings revealed that abrogation of EGFR signaling suppressed the increase in dermal neutrophils and mast cells following UV 
exposure. Since these recruited inflammatory cells release various cytokines and enzymes that enhance vascular permeability, suppression of such inflammatory cell infiltration would be expected to lessen the degree of inflammation. For example, Meyer-Hoffert et al. have found that EGFRneutralizing antibodies abolish the proliferative effect of human leukocyte elastase produced by neutrophils in human keratinocytes [43]. Moreover, reduction of infiltrating mast cells with their attendant degranulation in response to UV appears to alleviate inflammation, possibly through reduced release of mast cells specific products, such as histamine, TNF- $\alpha$, and the delayed phase of PGE2 synthesis mediated by COX-2 [28, 42]. In addition, mast cells are required for the melanocyte activation induced by endothelin-1 and, hence, the protective tanning response to UV [27].

In addition to the suppression of acute inflammation after UV exposure documented here, abrogation of EGFR function is also well known to lead to cutaneous inflammation, more specifically to folliculitis, in cancer patients undergoing longer-term treatment with EGFR inhibitors [44]. Thus, the role of EGFR in cutaneous inflammation is certainly complex. Because of the apparent multifaceted functions of EGFR in regulating cutaneous inflammation, further investigation into the role of EGFR-dependent pro- and anti-inflammatory effects is warranted.

\section{Conclusions}

Based on our data, we conclude that EGFR signaling contributed to UV-induced inflammation, potentially through multiple mechanisms. EGFR activation increased mast cell and neutrophil numbers in the skin, edema, and erythema, responses that may occur through both EGFR/p38 kinase/COX-2-dependent and independent mechanisms. Since we previously found that ErbB2 mediates skin inflammation in response to UV [45], examining the effects of combination therapies which target major activated receptor tyrosine kinases in skin following UV irradiation will prove interesting.

\section{Conflict of Interests}

Dr. Hansen's research is supported by the National Institutes of Health R01 ES015585 and the State of Nebraska LB595 Cancer and Smoking-Related Diseases Research Program. The authors Dr. El-Abaseri, Ms. Hammiller, and Dr. Repertinger have no conflict of interests to declare.

\section{Acknowledgments}

This research was supported by the National Institutes of Health (1RO1ES015585) and the State of Nebraska Cancer and Smoking-Related Diseases Research Program. This investigation was conducted in a facility constructed with support from Research Facilities Improvement Program Grant nos. 1CO6RR17417-01 and G20RR024001 from the National Center for Research Resources, National Institutes of Health. The content is solely the responsibility of the authors and does not necessarily represent the official views of the National Center for Research Resources or the National Institutes of Health. These funders played no role in study design, data collection, data analysis, paper preparation, or publication decisions.

\section{References}

[1] S. K. Repertinger, E. Campagnaro, J. Fuhrman, T. El-Abaseri, S. H. Yuspa, and L. A. Hansen, "EGFR enhances early healing after cutaneous incisional wounding," Journal of Investigative Dermatology, vol. 123, no. 5, pp. 982-989, 2004.

[2] A. Knebel, H. J. Rahmsdorf, A. Ullrich, and P. Herrlich, "Dephosphorylation of receptor tyrosine kinases as target of regulation by radiation, oxidants or alkylating agents," The EMBO Journal, vol. 15, no. 19, pp. 5314-5325, 1996.

[3] T. B. El-Abaseri, J. Fuhrman, C. Trempus, I. Shendrik, R. W. Tennant, and L. A. Hansen, "Chemoprevention of UV lightinduced skin tumorigenesis by inhibition of the epidermal growth factor receptor," Cancer Research, vol. 65, no. 9, pp. 3958-3965, 2005.

[4] T. B. El-Abaseri, S. Putta, and L. A. Hansen, "Ultraviolet irradiation induces keratinocyte proliferation and epidermal hyperplasia through the activation of the epidermal growth factor receptor," Carcinogenesis, vol. 27, no. 2, pp. 225-231, 2006.

[5] V. O. Melnikova and H. N. Ananthaswamy, "Cellular and molecular events leading to the development of skin cancer," Mutation Research, vol. 571, no. 1-2, pp. 91-106, 2005.

[6] G. J. Clydesdale, G. W. Dandie, and H. K. Muller, "Ultraviolet light induced injury: immunological and inflammatory effects," Immunology and Cell Biology, vol. 79, no. 6, pp. 547-568, 2001.

[7] J. H. Chung, J. Y. Seo, M. K. Lee et al., "Ultraviolet modulation of human macrophage metalloelastase in human skin in vivo," Journal of Investigative Dermatology, vol. 119, no. 2, pp. 507-512, 2002.

[8] C. S. Tripp, E. A. G. Blomme, K. S. Chinn, M. M. Hardy, P. LaCelle, and A. P. Pentland, "Epidermal COX-2 induction following ultraviolet irradiation: suggested mechanism for the role of COX-2 inhibition in photoprotection," Journal of Investigative Dermatology, vol. 121, no. 4, pp. 853-861, 2003.

[9] C. C. Miller, P. Hale, and A. P. Pentland, "Ultraviolet B injury increases prostaglandin synthesis through a tyrosine kinasedependent pathway. Evidence for UVB-induced epidermal growth factor receptor activation," Journal of Biological Chemistry, vol. 269, no. 5, pp. 3529-3533, 1994.

[10] S. Kondo, T. Kono, D. N. Sauder, and R. C. McKenzie, "Il-8 gene expression and production in human keratinocytes and their modulation by UVB," Journal of Investigative Dermatology, vol. 101, no. 5, pp. 690-694, 1993.

[11] M. Yoshizumi, T. Nakamura, M. Kato et al., "Release of cytokines/chemokines and cell death in UVB-irradiated human keratinocytes, HaCaT,' Cell Biology International, vol. 32, no. 11, pp. 1405-1411, 2008.

[12] I. Strickland, L. E. Rhodes, B. F. Flanagan, and P. S. Friedmann, "TNF- $\alpha$ and IL- 8 are upregulated in the epidermis of normal human skin after UVB exposure: correlation with neutrophil accumulation and E-selectin expression," Journal of Investigative Dermatology, vol. 108, no. 5, pp. 763-768, 1997.

[13] J. Krutmann and M. Grewe, "Involvement of cytokines, DNA damage, and reactive oxygen intermediates in ultraviolet radiation-induced modulation of intercellular adhesion molecule-1 expression," Journal of Investigative Dermatology, vol. 105, supplement 1, pp. 67S-70S, 1995. 
[14] D. Luo, M. Yaar, A. Tsai, and B. A. Gilchrest, "Solar-simulated irradiation evokes a persistent and biphasic IL-1 $\alpha$ response," Experimental Dermatology, vol. 13, no. 1, pp. 11-17, 2004.

[15] A. Kock, T. Schwarz, R. Kirnbauer et al., "Human keratinocytes are a source for tumor necrosis factor $\alpha$ : evidence for synthesis and release upon stimulation with endotoxin or ultraviolet light," Journal of Experimental Medicine, vol. 172, no. 6, pp.1609$1614,1990$.

[16] M. Wlaschek, G. Heinen, A. Poswig, A. Schwarz, T. Krieg, and K. Scharffetter-Kochanek, "UVA-induced autocrine stimulation of fibroblast-derived collagenase/MMP-1 by interrelated loops of interleukin-1 and interleukin-6," Photochemistry and Photobiology, vol. 59, no. 5, pp. 550-556, 1994.

[17] X.-Y. Wang and Z.-G. Bi, "UVB-irradiated human keratinocytes and interleukin- $1 \alpha$ indirectly increase MAP kinase/AP-1 activation and MMP-1 production in UVA-irradiated dermal fibroblasts," Chinese Medical Journal, vol. 119, no. 10, pp. 827-831, 2006.

[18] Y. Xu, Y. Shao, J. J. Voorhees, and G. J. Fisher, "Oxidative inhibition of receptor-type protein-tyrosine phosphatase $\kappa$ by ultraviolet irradiation activates epidermal growth factor receptor in human keratinocytes," Journal of Biological Chemistry, vol. 281, no. 37, pp. 27389-27397, 2006.

[19] A. L. Kim, J. M. Labasi, Y. Zhu et al., "Role of p38 MAPK in UVB-induced inflammatory responses in the skin of SKH-1 hairless mice," Journal of Investigative Dermatology, vol. 124, no. 6, pp. 1318-1325, 2005.

[20] S. Kondo, "The roles of cytokines in photoaging," Journal of Dermatological Science, vol. 23, supplement 1, pp. S30-S36, 2000.

[21] W. Chen, Q. Tang, M. S. Gonzales, and G. T. Bowden, "Role of p38 MAP kinases and ERK in mediating ultraviolet-B induced cyclooxygenase-2 gene expression in human keratinocytes," Oncogene, vol. 20, no. 29, pp. 3921-3926, 2001.

[22] M. Ashida, T. Bito, A. Budiyanto, M. Ichihashi, and M. Ueda, "Involvement of EGF receptor activation in the induction of cyclooxygenase-2 in HaCaT keratinocytes after UVB," Experimental Dermatology, vol. 12, no. 4, pp. 445-452, 2003.

[23] U. Lichti, J. Anders, and S. H. Yuspa, "Isolation and short-term culture of primary keratinocytes, hair follicle populations and dermal cells from newborn mice and keratinocytes from adult mice for in vitro analysis and for grafting to immunodeficient mice," Nature Protocols, vol. 3, no. 5, pp. 799-810, 2008.

[24] D. W. Threadgill, A. A. Dlugosz, L. A. Hansen et al., "Targeted disruption of mouse EGF receptor: effect of genetic background on mutant phenotype," Science, vol. 269, no. 5221, pp. 230-234, 1995.

[25] K. Yano, K. Kadoya, K. Kajiya, Y.-K. Hong, and M. Detmar, "Ultraviolet B irradiation of human skin induces an angiogenic switch that is mediated by upregulation of vascular endothelial growth factor and by downregulation of thrombospondin-1," British Journal of Dermatology, vol. 152, no. 1, pp. 115-121, 2005.

[26] S. Guhl, R. Stefaniak, M. Strathmann et al., "Bivalent effect of UV light on human skin mast cells-low-level mediator release at baseline but potent suppression upon mast cell triggering," Journal of Investigative Dermatology, vol. 124, no. 2, pp. 453-456, 2005.

[27] M. Metz, V. Lammel, B. F. Gibbs, and M. Maurer, "Inflammatory murine skin responses to UV-B light are partially dependent on endothelin-1 and mast cells," American Journal of Pathology, vol. 169, no. 3, pp. 815-822, 2006.
[28] L. J. Walsh, "Ultraviolet B irradiation of skin induces mast cell degranulation and release of tumour necrosis factor- $\alpha$," Immunology and Cell Biology, vol. 73, no. 3, pp. 226-233, 1995.

[29] T. A. Wilgus, M. L. Parrett, M. S. Ross, K. L. Tober, F. M. Robertson, and T. M. Oberyszyn, "Inhibition of ultraviolet light Binduced cutaneous inflammation by a specific cyclooxygenase2 inhibitor," Advances in Experimental Medicine and Biology, vol. 507, pp. 85-92, 2002.

[30] D. M. Ouwens, N. D. de Ruiter, G. C. M. van der Zon et al., "Growth factors can activate ATF2 via a two-step mechanism: phosphorylation of Thr71 through the Ras-MEK-ERK pathway and of Thr69 through RaIGDS-Src-p38," The EMBO Journal, vol. 21, no. 14, pp. 3782-3793, 2002.

[31] R. M. Hobbs and F. M. Watt, "Regulation of interleukin- $1 \alpha \mathrm{a}$ expression by integrins and epidermal growth factor receptor in keratinocytes from a mouse model of inflammatory skin disease," Journal of Biological Chemistry, vol. 278, no. 22, pp. 19798-19807, 2003.

[32] S. Pastore, F. Mascia, F. Mariotti, C. Dattilo, V. Mariani, and G. Girolomoni, "ERK1/2 regulates epidermal chemokine expression and skin inflammation," Journal of Immunology, vol. 174, no. 8, pp. 5047-5056, 2005.

[33] J. Hildesheim, R. T. Awwad, and A. J. Fornace Jr., "p38 Mitogenactivated protein kinase inhibitor protects the epidermis against the acute damaging effects of ultraviolet irradiation by blocking apoptosis and inflammatory responses," Journal of Investigative Dermatology, vol. 122, no. 2, pp. 497-502, 2004.

[34] T. A. Wilgus, A. T. Koki, B. S. Zweifel, D. F. Kusewitt, P. A. Rubal, and T. M. Oberyszyn, "Inhibition of cutaneous ultraviolet light B-mediated inflammation and tumor formation with topical celecoxib treatment," Molecular Carcinogenesis, vol. 38, no. 2, pp. 49-58, 2003.

[35] S. Trompezinski, I. Pernet, D. Schmitt, and J. Viac, "UV radiation and prostaglandin E2 up-regulate vascular endothelial growth factor (VEGF) in cultured human fibroblasts," Inflammation Research, vol. 50, no. 8, pp. 422-427, 2001.

[36] T. A. Wilgus, A. T. Koki, B. S. Zweifel, P. A. Rubal, and T. M. Oberyszyn, "Chemotherapeutic efficacy of topical celecoxib in a murine model of ultraviolet light B-induced skin cancer," Molecular Carcinogenesis, vol. 38, no. 1, pp. 33-39, 2003.

[37] G. J. Fisher, S. Kang, J. Varani et al., "Mechanisms of photoaging and chronological skin aging," Archives of Dermatology, vol. 138, no. 11, pp. 1462-1470, 2002.

[38] K. P. An, M. Athar, X. Tang et al., "Cyclooxygenase-2 expression in murine and human nonmelanoma skin cancers: implications for therapeutic approaches," Photochemistry and Photobiology, vol. 76, no. 1, pp. 73-80, 2002.

[39] J. E. Rundhaug, C. Mikulec, A. Pavone, and S. M. Fischer, "A role for cyclooxygenase-2 in ultraviolet light-induced skin carcinogenesis," Molecular Carcinogenesis, vol. 46, no. 8, pp. 692-698, 2007.

[40] A. Johnston, J. E. Gudjonsson, A. Aphale, A. M. Guzman, S. W. Stoll, and J. T. Elder, "EGFR and IL-1 signaling synergistically promote keratinocyte antimicrobial defenses in a differentiation-dependent manner," Journal of Investigative Dermatology, vol. 131, no. 2, pp. 329-337, 2011.

[41] F. Mascia, C. Cataisson, T.-C. Lee et al., "EGFR regulates the expression of keratinocyte-derived granulocyte/macrophage colony-stimulating factor in vitro and in vivo," Journal of Investigative Dermatology, vol. 130, no. 3, pp. 682-693, 2010.

[42] S. T. Reddy, H. F. Tiano, R. Langenbach, S. G. Morham, and H. R. Herschman, "Genetic evidence for distinct roles of 
COX-1 and COX-2 in the immediate and delayed phases of prostaglandin synthesis in mast cells," Biochemical and Biophysical Research Communications, vol. 265, no. 1, pp. 205-210, 1999.

[43] U. Meyer-Hoffert, J. Wingertszahn, and O. Wiedow, "Human leukocyte elastase induces keratinocyte proliferation by epidermal growth factor receptor activation," Journal of Investigative Dermatology, vol. 123, no. 2, pp. 338-345, 2004.

[44] M. E. Lacouture, "Mechanisms of cutaneous toxicities to EGFR inhibitors," Nature Reviews Cancer, vol. 6, no. 10, pp. 803-812, 2006.

[45] J. G. Madson, D. T. Lynch, K. L. Tinkum, S. K. Putta, and L. A. Hansen, "Erbb2 regulates inflammation and proliferation in the skin after ultraviolet irradiation," American Journal of Pathology, vol. 169, no. 4, pp. 1402-1414, 2006. 


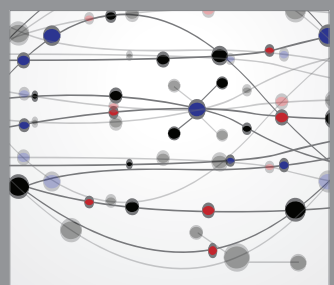

The Scientific World Journal
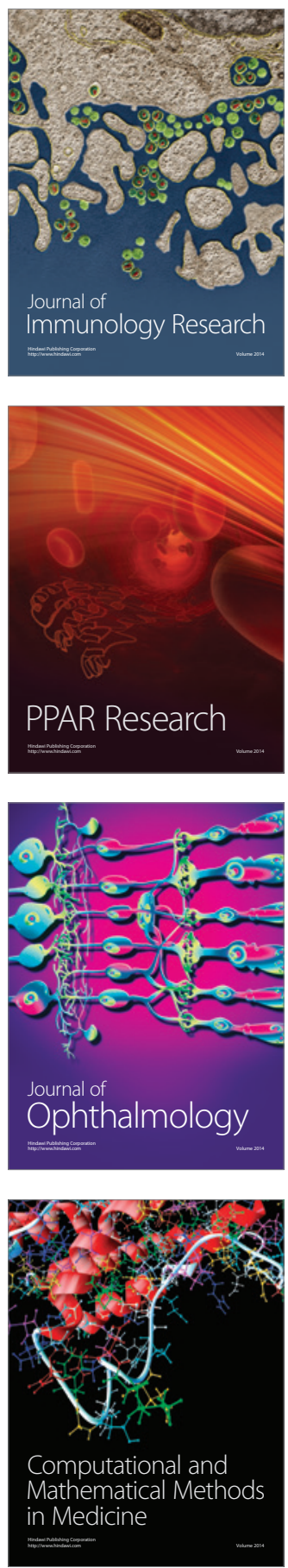

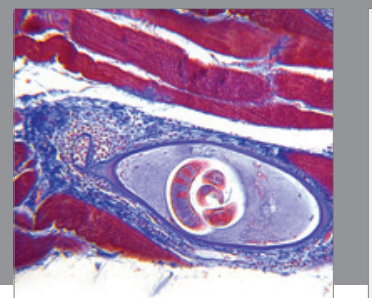

Gastroenterology

Research and Practice
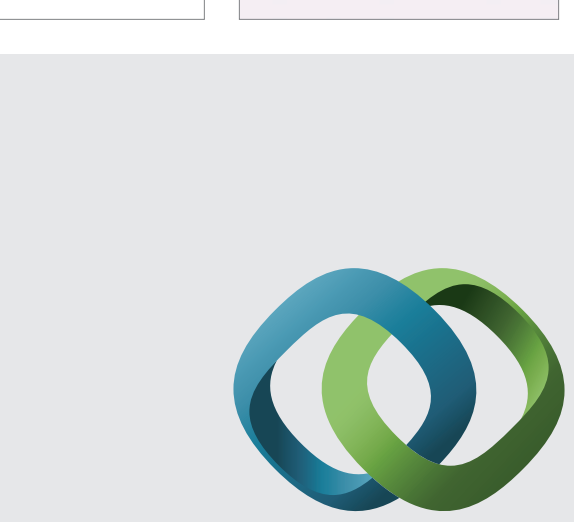

\section{Hindawi}

Submit your manuscripts at

http://www.hindawi.com
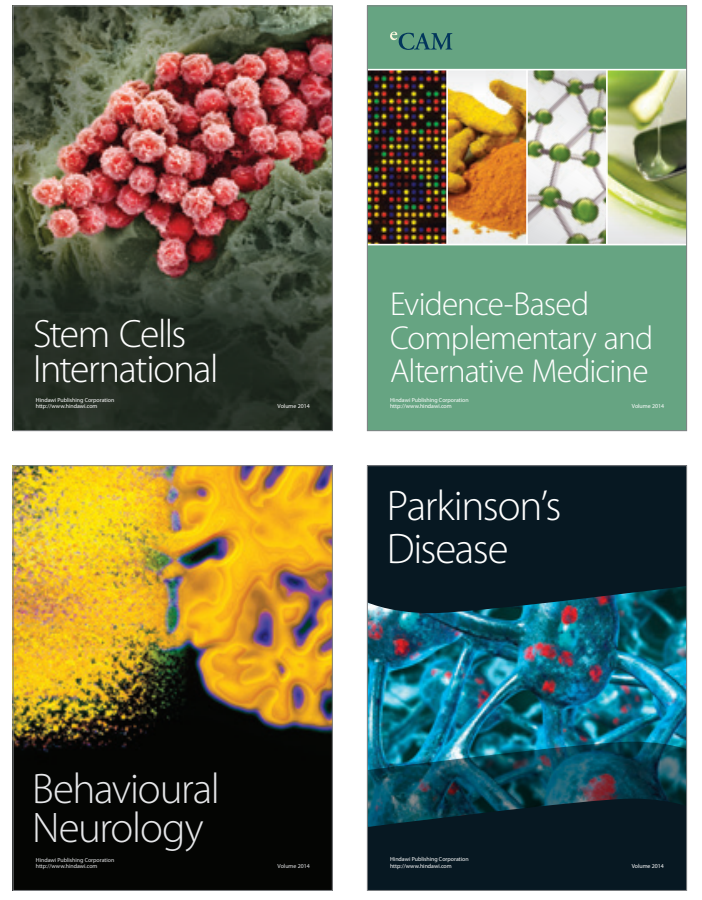
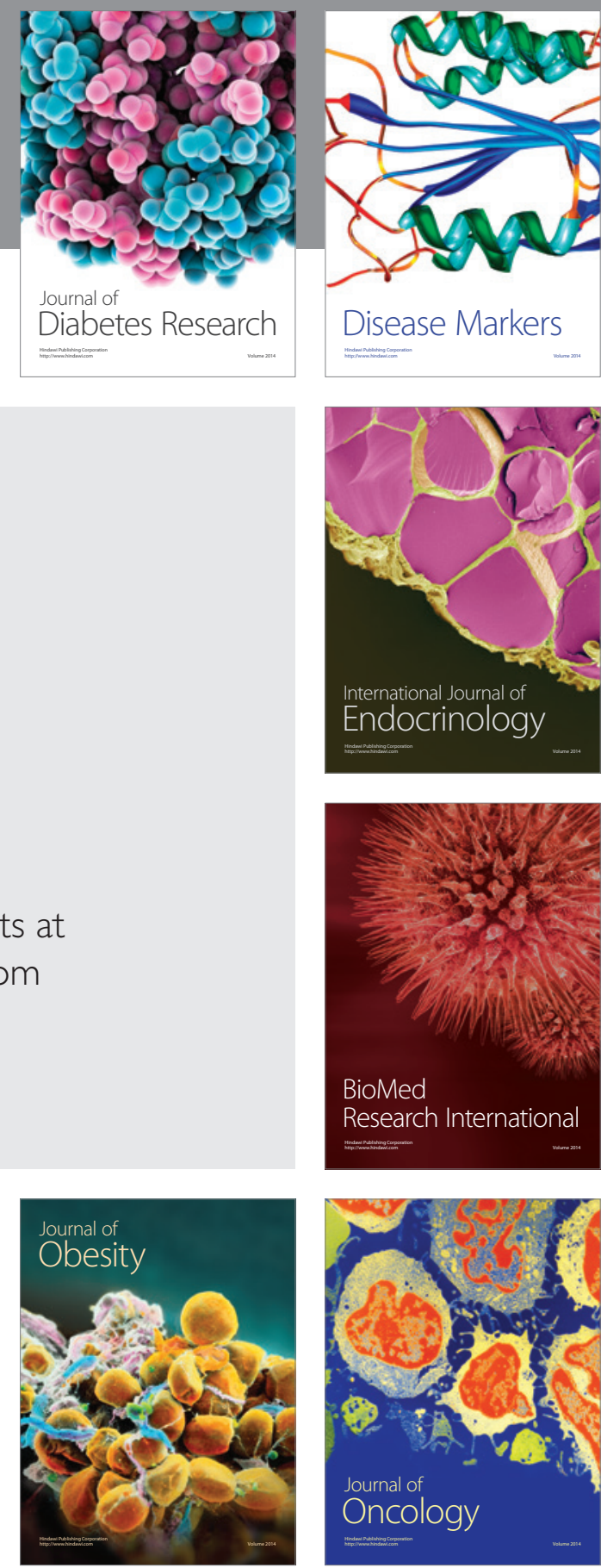

Disease Markers
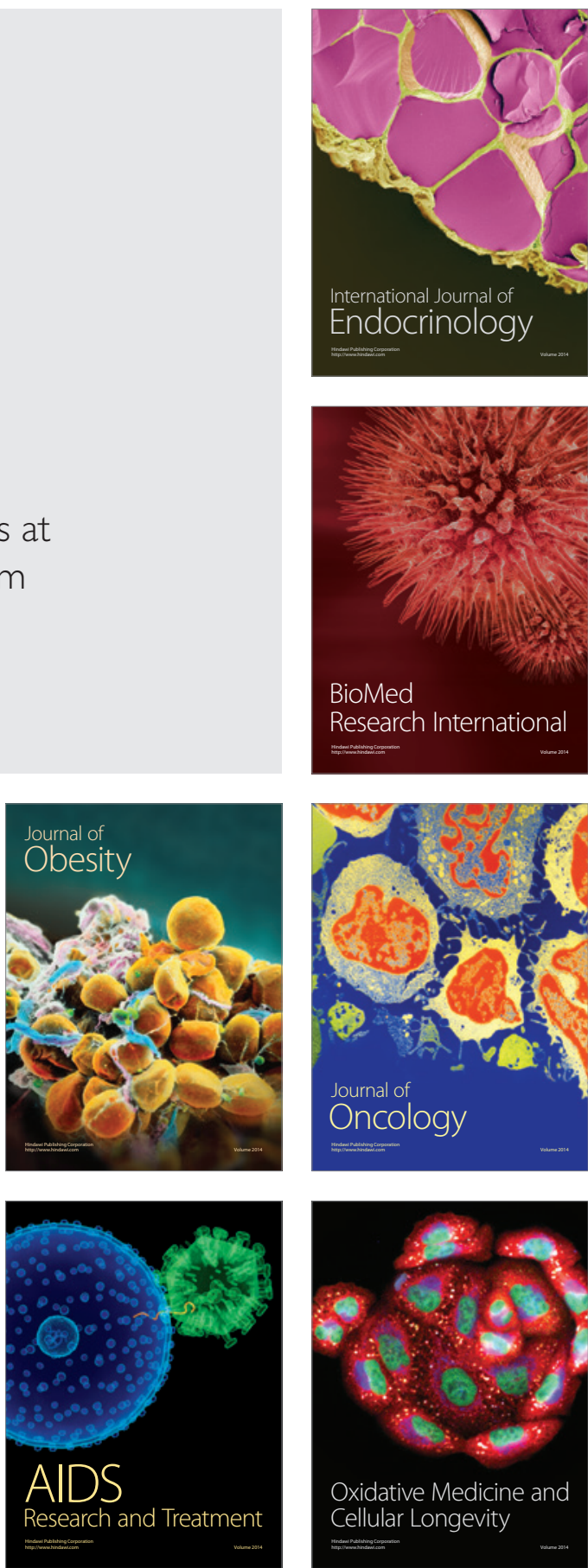